\title{
Pulmonary complications and survival after autologous stem cell transplantation: predictive role of pulmonary function and pneumotoxic medications
}

To the Editor:

Autologous stem cell transplantation (ASCT) is the standard of care for multiple myeloma patients eligible for high-dose therapy, lymphoma patients undergoing second-line treatments and for acute myelogenous leukaemia (AML) [1]. Immune system impairment and chemotherapies significantly increase the risk of infections, particularly pneumonia [2]. Overall, pulmonary complications, both infectious and non-infectious, occur in $40-60 \%$ of patients after stem cell transplantation [3], and are usually classified as early or late onset, depending on whether they occur within 100 days of the transplant [4]. The underlying disease and baseline pulmonary function, along with conditioning regimens consisting of carmustine, etoposide, aracytin and melphalan for lymphoma, melphalan alone for multiple myeloma or busulpan and cyclophosphamide for acute myeloid leukaemia, all concur to cause pulmonary complications $[3,4]$.

Many reports focus on allogenic transplantation alone [5], but the only report dealing with ASCT displayed a $25 \%$ prevalence of pulmonary complications within the first year post-ASCT and a severe associated risk of death [6].

The aim of our study was to assess whether baseline pulmonary function tests help to define the risk of pulmonary complications/adverse events or death after ASCT, and determine whether pneumotoxic induction treatment affects this risk.

No ethics committee approval was required as this was an observational study. Nonetheless, all patients signed an informed consent form. Between March 2008 and August 2015, we prospectively analysed 81 consecutive patients eligible for ASCT for haematological diseases. First-line treatment with immunomodulatory drugs, proteasome inhibitors and dexamethasone for multiple myeloma, and anthracycline-based protocols for lymphomas, followed by mobilisation therapy (favouring stem cell release into peripheral blood) with a high dose of cyclophosphamide was carried out. All patients underwent complete laboratory assessments, total-body computed tomography (CT) scans, and cardiac and pulmonary function tests. Baseline comorbidity was scored by the ASCT tailored Sorror score [7]. Exposure to pneumotoxic medications during the induction treatment was recorded, and lung abnormalities were identified by CT imaging. Any infective complications or pulmonary events were also recorded [8].

We reported early and late respiratory infections and causes of death, generating different survival curves. The study outcomes were overall survival, the time between the start of treatment and death from any cause; event-free survival, the time between the start of treatment and recurrence or death for any cause; infection-free interval, the time between the start of treatment and the first infective event.

Treatment response was categorised as partial or complete, stable disease or disease progression [8].

Spirometry and flow-volume curves were obtained according to current guidelines [9]. Residual volume and total lung capacity (TLC) were measured using the helium dilution technique.

Diffusing capacity of the lung for carbon monoxide (DLCO), expressed in $\mathrm{mL} \cdot \mathrm{min}^{-1} \cdot \mathrm{mmHg}^{-1}$, was obtained by a single-breath method using a dedicated gas chromatography system (Biomedin, Padua, Italy).

@ERSpublications

Pneumotoxic agents prior to ASCT associate with worse event/infection free survival and overall mortality http://ow.ly/1xkL308x3D2

Cite this article as: Scarlata S, Annibali O, Santangelo S, et al. Pulmonary complications and survival after autologous stem cell transplantation: predictive role of pulmonary function and pneumotoxic medications. Eur Respir J 2017; 49: 1601902 [https://doi.org/10.1183/13993003.01902-2016]. 
Anti-infective prophylactic therapy conformed to the proposed guidelines [10]. No invasive fungal infections occurred before ASCT. Weekly surveillance of cytomegalovirus reactivation and serum galactomannan assay were also performed [11]. In case of clinically suspected infection, after specimen collection for microbiology studies (blood, sputum, urine and swabs of the orifices), first-line empiric antibiotic treatment was initiated [12]. A chest CT scan was performed on all patients with fever and neutropenia. Bronchoscopy and bronchoalveolar lavage were performed only when there was no clinical response or conditions worsened despite the antibiotic therapy [13].

Bacterial, viral, fungal and Pneumocystis jirovecii pneumonias were determined according to available guidelines [10].

Groups were compared using the $\chi$-square test for categorical variables, and one-way ANOVA tests followed by the Bonferroni multiple comparison post-hoc adjustment for continuous variables. Values were expressed as mean, median, 95\% confidence interval for standard error of means (SEM) and standard deviation (SD). Kaplan-Meier curves and proportional hazards models were used to assess the relationships between lung function, pneumotoxic agent exposure and post-transplant survival, as well as IFI. All statistical analyses were performed using SPSS software (version 19.0; SPSS Inc., Chicago, IL, USA).

The mean age of patients was 53.7 years, with a SEM of 10.3 years; $67.9 \%$ of these had multiple myeloma. Remarkably, 39 out of 81 patients (48.1\%) were treated with potentially pneumotoxic agents (bleomycin, bortezomib, thalidomide or lenalidomide) and 19 out of $81(23.5 \%)$ presented lung abnormalities on a chest CT scan. The response to induction treatments was partial in $64.2 \%$, complete in $28 \%$ and absent in $7.8 \%$.

After a median (range) follow up of 17.3 months (1-85 months), 30 out of 81 patients (37\%) died because of disease progression (13/30); infective complications (7/30); lung cancer (1/30); cerebral haemorrhage $(1 / 30)$; other causes $(8 / 30)$. Major infective episodes, including 12 early and eight late onset respiratory infections, affected 43 out of 81 patients $(53.1 \%)$

At baseline, lower values of the forced expiratory volume in $1 \mathrm{~s}$ (FEV1) $(92.7 \pm 16.8 \%$ versus $101.6 \pm 16.2 \%$; $\mathrm{p}=0.018)$, forced vital capacity (FVC) $(95.5 \pm 15.4 \%$ versus $104.9 \pm 16.2 \% ; \mathrm{p}=0.009)$ and TLC $(89.4 \pm 13.5 \%$ versus $96.3 \pm 14.0 \%$; $\mathrm{p}=0.05)$ were observed in patients exposed to pneumotoxic agents, and greater values of FEV1 (92.4 $\pm 18.6 \%$ versus $101.2 \pm 15.4 \%$; $\mathrm{p}=0.03)$, FVC $(95.6 \pm 15.9 \%$ versus $104.2 \pm 16.0 \%$; $=0.03)$ and carbon monoxide transfer coefficient $(\mathrm{KCO})(2.52 \pm 0.8$ versus $3.22 \pm 1.1 ; \mathrm{p}=0.03)$ were recorded in survivors. Though statistically significant, these differences were clinically negligible, given that only 14 out of the 81 patients had a mildly abnormal spirometric pattern. The lung function values expressed as mean \pm sD explained the overlap in the $95 \%$ confidence intervals $( \pm 2 \mathrm{SD}$ ) between the two groups tested despite the given p-values being significant at the 0.05 level as the statistical tests referred to would have used SEM to test for differences in the mean between the two groups. This finding, though not uncommon, still needs to be highlighted.

Overall survival, event-free survival and infection-free interval curves are shown in figure 1 . The exposed group had a median overall survival of 40.0 versus $>60$ months for the non-exposed population $(p=0.01)$ (figure 1a), with a median event-free survival of 2 versus 52.6 months $(\mathrm{p}=0.004)$ (figure $1 \mathrm{~b})$, and a median infection-free interval of 1.2 versus 58.5 months ( $\mathrm{p}=0.028$ ) (figure $1 \mathrm{c}$ ).

Pre-ASCT mean FEV1, FVC and KCO were significantly lower in patients who died, but no difference in the infection rate was observed. Overall survival curves by FEV1and FVC did not differ among exposed and non-exposed patients. Instead, a longer overall survival by DLCO $(p=0.05)$ and KCO $(p=0.04)$ characterised the unexposed cohort.

Pulmonary function testing did not distinguish ASCT recipients who had an infective event, an early or a late onset respiratory infection from those who remained free from infections.

This is the first longitudinal design study to show that pre-transplant pulmonary function tests and exposure to pneumotoxic agents during the induction therapy help to predict mortality and infection-free interval following ASCT. In fact, patients exposed to pneumotoxic agents before ASCT experienced significantly worse overall survival, event-free survival and infection-free interval. Specifically, infections (mainly non-respiratory) and early non-infectious adverse events had a heavy prognostic impact, the median infection-free interval being 1.2 months in the exposed group and 58.5 months in the non-exposed group.

Another main finding of our study was that some baseline respiratory mean values (FEV1, FVC and TLC) were statistically lower in patients previously exposed to potentially pneumotoxic agents, and such reduction predicts worse outcomes. BRUCE et al. [14] found an increased likelihood of developing bronchial obstruction and a restrictive spirometric pattern in multiple myeloma subjects exposed to bortezomib following thalidomide, but survival data were not provided. Previous reports on the relationship between stem cell transplantation and pre-transplant abnormal pulmonary function tests refer to mixed populations of allogenic and autologous recipients [15], making the results difficult to interpret. 

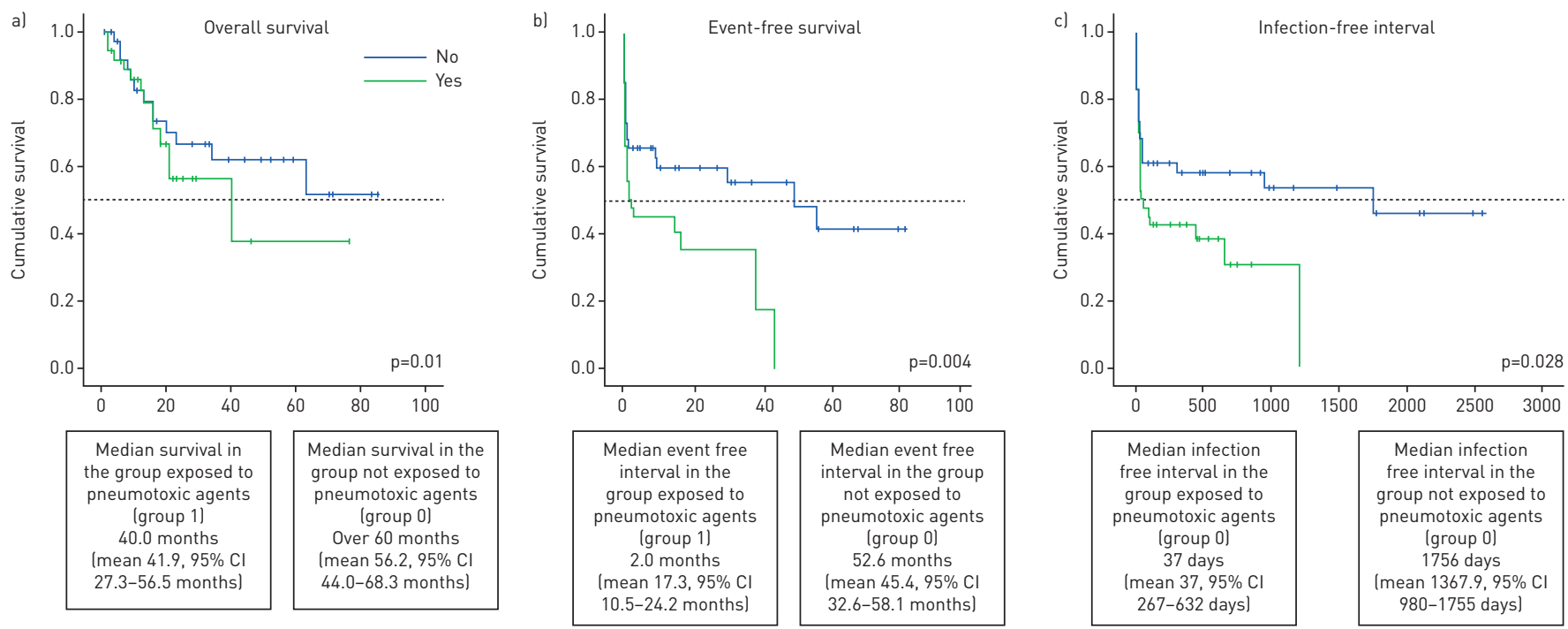

FIGURE 1 a) Overall survival, b) event-free survival and c) infection-free interval curves observed in the two groups exposed or not to pneumotoxic drugs.

Interestingly, in our population, a lower respiratory volume and lung diffusion index predicted worse overall survival and shorter infection-free interval more accurately than other recognised prognostic indexes, such as the Sorror comorbidity score.

If confirmed, it may be relevant that baseline respiratory conditions complement other well-recognised indicators to identify the risk of complications in ASCT candidates. Because of the single centre design and the small sample size make our data are preliminary data. Finally, we failed to test the association between pneumotoxic medication exposure and lung function decline following the ASCT.

In conclusion, exposure to pneumotoxic agents before ASCT increases the risk of pulmonary function abnormalities and to affect infection-free interval and event-free survival, as well as overall survival. If confirmed by a larger, multicentre study, these findings could influence pre-transplant assessment and management.

Simone Scarlata ${ }^{1,4}$, Ombretta Annibali ${ }^{2,4}$, Simona Santangelo ${ }^{1}$, Valeria Tomarchio $^{2}$, Silvia Ferraro ${ }^{2}$, Daniele Armiento ${ }^{2}$, Alessandra Scardocci ${ }^{2}$, William Arcese ${ }^{3}$, Raffaele Antonelli Incalzi ${ }^{1}$ and Giuseppe Avvisati ${ }^{2}$

${ }^{1}$ Geriatrics - Dept of Respiratory Pathophysiology and Thoracic Endoscopy, Campus Bio Medico University and Teaching Hospital, Rome, Italy. ${ }^{2}$ Dept of Stem Cell Transplantation, Transfusion Medicine and Cellular Therapy, Campus Bio Medico University and Teaching Hospital, Rome, Italy. ${ }^{3}$ Dept of Stem Cell Transplantation, Rome Transplant Network, Dept of Haematology, "Tor Vergata" University Hospital, Rome, Italy. ${ }^{4}$ Both authors contributed equally.

Correspondence: Simone Scarlata, Campus Bio Medico University and Teaching Hospital, Via Alvaro del Portillo 200-00128 Rome, Italy. E-mail: s.scarlata@unicampus.it

Received: July 032016 | Accepted after revision: Dec 152016

Conflict of interest: None declared.

\section{References}

1 Majhail NS, Farnia SH, Carpenter PA, et al. Indications for autologous and allogeneic hematopoietic cell transplantation: Guidelines from the American Society for Blood and Marrow Transplantation. Biol Blood Marrow Transplant 2015; 21: 1863-1869.

2 Dichter JR, Levine SJ, Shelhamer JH. Approach to the immunocompromised host with pulmonary symptoms. Hematol Oncol Clin North Am 1993; 7: 887-912.

3 Kotloff RM, Ahya VN, Crawford SW. Pulmonary complications of solid organ and hematopoietic stem cell transplantation. Am J Respir Crit Care Med 2004; 170: 22-48.

4 Rossi SE, Erasmus JJ, McAdams HP, et al. Pulmonary drug toxicity: radiologic and pathologic manifestations. Radiographics 2000; 20: 1245-1259.

5 Lee MY, Chiou TJ, Yang MH, et al. Relatively favorable outcomes of post-transplant pulmonary function in patients with chronic myeloid leukemia receiving non-myeloablative allogeneic hematopoietic stem cell transplantation. Eur J Haematol 2005; 74: 152-157.

6 Afessa B, Abdulai RM, Kremers WK, et al. Risk factors and outcome of pulmonary complications after autologous hematopoietic stem cell transplant. Chest 2012; 141: 442-450.

7 Sorror ML. How I assess comorbidities before hematopoietic cell transplantation. Blood 2013; 121: 2854-2863.

8 Paiva B, van Dongen JJ, Orfao A. New criteria for response assessment: role of minimal residual disease in multiple myeloma. Blood 2015; 125: 3059-3068. 
9 Standardized lung function testing. Official statement of the European Respiratory Society. Eur Respir J 1993; 6: Suppl. 16, 1-100.

10 Yokoe D, Casper C, Dubberke E, et al. Infection prevention and control in health-care facilities in which hematopoietic cell transplant recipients are treated. Bone Marrow Transplant 2009; 44: 495-507.

11 Girmenia C, Aversa F, Busca A, et al. A hematology consensus agreement on antifungal strategies for neutropenic patients with hematological malignancies and stem cell transplant recipients. Gruppo Italiano Malattie Ematologiche dell'Adulto, Gruppo Italiano Trapianto di Midollo Osseo, Associazione Italiana Ematologia ed Oncologia Pediatrica, Invasive Fungal Infections Cooperative Group of the European Organization for Research and Treatment of Cancer and Sorveglianza Epidemiologica delle Infezioni Fungine nelle Emopatie Maligne. Hematol Oncol 2013; 31: 117-126.

12 Jing Y, Li J, Yuan L, et al. Piperacillin-tazobactam vs. imipenem-cilastatin as empirical therapy in hematopoietic stem cell transplantation recipients with febrile neutropenia. Clin Transplant 2016; 30: 263-269.

13 Harris B, Lowy FD, Stover DE, et al. Diagnostic bronchoscopy in solid-organ and hematopoietic stem cell transplantation. Ann Am Thorac Soc 2013; 10: 39-49.

14 Bruce JT, Tran JM, Phillips G, et al. Chemotherapeutic agents increase the risk for pulmonary function test abnormalities in patients with multiple myeloma. Clin Lymphoma Myeloma Leuk 2012; 12: 325-329.

15 Chien JW, Madtes DK, Clark JG. Pulmonary function testing prior to hematopoietic stem cell transplantation. Bone Marrow Transplant 2005; 35: 429-435. 